Supporting Information

\title{
Enhanced Electron Transfer Kinetics of Covalent Carbon
}

\section{Nanotube Junctions}

Sriram Kumar ${ }^{1,2, \$}$, Sumit Bawari ${ }^{3, \$}$, Sreekanth Narayanaru ${ }^{3, \$}$, Tharangattu N. Narayanan ${ }^{3 *}$, Ashis Kumar Satpati ${ }^{1,2^{*}}$

${ }^{1}$ Analytical Chemistry Division, Bhabha Atomic Research Centre, Trombay, Mumbai400094, India

${ }^{2}$ Homi Bhabha National Institute, Anuskatinagar, Mumbai-400094, India

${ }^{3}$ Tata Institute of Fundamental Research-Hyderabad, Hyderabad, 500046, India

*Corresponding author: T. N. Narayanan (tnn@tifrh.res.in) and A.K.Satpati

(asatpati@barc.gov.in)

$\left({ }^{\$}\right.$ Equal contribution)

\section{EXPERIMENTAL METHOD}

1.1. Synthesis. A detailed synthesis protocol for the development of coupled CNT (3DCNTs) is given elsewhere. ${ }^{1}$ In a nutshell, MWCNTs (200 mg), purchased from SigmaAldrich, were functionalized through treatment in conc. $\mathrm{HNO}_{3}(40 \mathrm{ml})$ for 48 hours at $110^{\circ}$ C. The oxidized CNTs $\left(90 \mathrm{mg}\right.$ ) obtained were chlorinated via $\mathrm{SOCl}_{2}(35 \mathrm{ml})$, where 1,2 
dichloro-benzene $(5 \mathrm{ml})$ acts as a mixing phase. The CNT-Cl $(20 \mathrm{mg})$ obtained was linked through Suzuki coupling, with the linker 1,4 diboronic-benzene $(10 \mathrm{mg})$ in toluene $(30 \mathrm{ml})$, and $\mathrm{CsCO}_{3}(160 \mathrm{mg})$ and $\mathrm{Pd}\left(\mathrm{PPh}_{3}\right)_{4}(30 \mathrm{mg})$ act as catalysts. As the functional group $\mathrm{C}(\mathrm{O})-\mathrm{Cl}$ is more labile in comparison to $\mathrm{C}-\mathrm{Cl}$ during the coupling reaction, $\mathrm{C}-\mathrm{Cl}$ has been considered as the primary coupling intermediate. ${ }^{2}$ The coupled CNT (3D-CNT) obtained was washed with diluted aqua regia and water to remove excess metals.

1.2. Characterization. SEM imaging was performed using a Jeol JSM7200F model SEM, at $15 \mathrm{kV}$ acceleration. Raman spectra was taken using Renishaw in Via micro-Raman spectrometer, with a $532 \mathrm{~nm}$ laser (100× objective lens and $20 \mathrm{~s}$ acquisition at $5 \%$ intensity).

1.3. Electrochemical measurements. All electrochemical studies were carried out at room temperature using $920 \mathrm{D}$ bipotentiostat $(\mathrm{CH}$ Instrument, Texas) in three electrodes configuration. $\mathrm{Ag} / \mathrm{AgCl}(3 \mathrm{M} \mathrm{KCl})$ was used as reference electrode and $\mathrm{Pt}$ wire as the counter electrode. Modified $3 \mathrm{~mm}$ glassy carbon electrode (GCE) was used as working electrode. Prior modification, GCE was polished with alumna powder of size $0.3 \mu \mathrm{m}$ and $0.05 \mu \mathrm{m}$ and then sonicated in $50 \%$ ethanol to remove surface impurities. CNT (3D-CNT and commercial CNT) inks were coated on IR lamp dried GCE for electrochemical measurements. The homogeneous catalyst inks were prepared by sonication of $2 \mathrm{mg}$ of catalysts in $1 \mathrm{~mL}$ of water-isopropyl alcohol solution (3:1). 1\% of Nafion solution (Sigma Aldrich, 5\%) was added into catalyst ink. After that, $5 \mu \mathrm{L}$ of the catalyst ink was drop-casted on GCE and dried in IR lamp. The geometric surface area of GCE was $\sim 0.07 \mathrm{~cm}^{2}$ and loading of catalyst on electrode was $142 \mu \mathrm{g} / \mathrm{cm}^{2}$ (loading of catalyst was same in all electrochemical studies). Prior to electrochemical studies, catalysts were preconditioned by running cyclic voltammetry (CV) in $0.1 \mathrm{M}$ PBS (pH 7) for 50 cycles from $0.5 \mathrm{~V}$ to $-0.5 \mathrm{~V}$ (vs $\mathrm{Ag} / \mathrm{AgCl}$ ).

The SECM study was performed using four electrodes systems. The modified glassy carbon 
electrode (GCE) was used as the substrate, $\mathrm{Ag} / \mathrm{AgCl}(3 \mathrm{M} \mathrm{KCl})$ as the reference electrode and Pt wire as the counter electrode. Homemade electrochemical cell was used for SECM experiment in which modified GCE (substrate) was inserted in inverted mode. The Pt- ultramicroelectrode (UME) of RG 5 and diameter $10 \mu \mathrm{m}$ was used as the probe. Prior to the use of the probe, UME was polished with a micro polishing cloth using alumina powder of size 0.30 $\mu \mathrm{m}$ and $0.05 \mu \mathrm{m}$ subsequently followed by electrochemical cleaning in $0.50 \mathrm{M} \mathrm{H}_{2} \mathrm{SO}_{4}$ solution. UME was cleaned by performing cyclic voltammetry $(\mathrm{CV})$ in a potential window of $0.80 \mathrm{~V}$ to $-0.35 \mathrm{~V}$ for 20 cycles. $5 \mathrm{mM}\left[\mathrm{Fe}(\mathrm{CN})_{6}\right]^{4-}$ in $0.1 \mathrm{M}$ PBS (pH 7) was used as the redox probe. Pt-UME was characterized by recording $\mathrm{CV}$ in $5 \mathrm{mM}\left[\mathrm{Fe}(\mathrm{CN})_{6}\right]^{4-}$ solution as shown the Figure $\mathrm{S} 1 \mathrm{~b}$. The $\mathrm{CV}$ response shows the excellent charge transfer over the UME and very less capacitive property. The size of the UME was calculated using the following equation (1)

$$
I_{d}=4 n F D C a
$$

Where $I_{d}$ is diffusion-limited current, $\mathrm{n}$ is the number of electrons transferred at the tip (probe), $\mathrm{F}$ is Faraday's constant, $\mathrm{D}$ is the diffusion coefficient of $\mathrm{Fe}^{2+}\left(6.7 \times 10^{-6} \mathrm{~cm}^{2} / \mathrm{s}\right)^{3}, \mathrm{C}$ is the concentration of $\mathrm{Fe}^{2+}(5 \mathrm{mM})$ and a is the radius of the UME disc. The diameter of the UME was obtained as $\sim 10 \mu \mathrm{m}$ from the steady-state current measurements.

Prior to SECM experiment, substrate was tilt corrected using three-point correction. Probe was approached to the substrate using probe approach curve technique (PAC) in $5 \mathrm{mM}$ ferrocyanide solution. Approach of probe was done at five different points to measure surface heterogeneity. Surface of catalyst was homogeneous with $\pm 5 \mu \mathrm{m}$ roughness as measured with PAC. Interfacial charge transfer kinetics was measured by probe approach curve at different substrate polarization potential at five different points. Charge transfer rate constant 
was calculated at electrode-electrolyte interface by numerical fitting of approach curve using following equations 4

$$
I_{T}^{i n s}(L, R G)=\frac{\frac{2.08}{R G^{0.358}}\left(L-\frac{0.145}{R G}\right)+1.585}{\frac{2.08}{R G^{0.358}}(L+0.0023 R G)+1.57+\frac{L n R G}{L}+\frac{2}{\pi R G} \operatorname{Ln}\left(1+\frac{\pi R G}{2 L}\right)}
$$

$I_{T}^{\text {cond }}\left(L+\kappa^{-1}, R G\right)$

$$
\begin{gathered}
=\alpha(R G)+\frac{\pi}{4 \beta(R G) \arctan \left(L+\kappa^{-1}\right)}+\left(1-\alpha(R G)-\frac{1}{2 \beta(R G)}\right) \frac{2}{\pi} \arctan \left(L+\kappa^{-1}\right) \\
I_{T}(L, \kappa, R G)=I_{T}^{\text {cond }}\left(L+\frac{1}{\kappa}, R G\right)+\frac{I_{T}^{\text {ins }}(L, R G)-1}{\left(1+2.47 R G^{0.31} L \kappa\right)\left(1+L^{0.006 R G+0.113} \kappa^{-0.023 R G+0.91}\right.} \\
I_{T}=I_{S}\left(1-\frac{I_{T}^{\text {ins }}}{I_{T}^{\text {cond }}}\right)+I_{T}^{\text {ins }} \\
k_{\text {eff }}=\kappa \frac{D_{\text {diffusion }}}{r_{T}}
\end{gathered}
$$

Where $I_{T}^{\text {ins }}$ is diffusion control current for insulating substrate i.e. negative feedback, $I_{T}^{\text {cond }}$ is diffusion control current for conducting substrate, i.e. positive feedback, $I_{T}$ total current for modified electrode, $R G=r_{\text {glass }} / r_{T}$, where $r_{\text {glass }}$ is radius of glass sheet, $r_{T}$ is radius of UME. After approach, SECM mapping of substrate was done at constant height mode at 20 $\mu \mathrm{m}$ from the substrate.

1.4. DFT Calculations. The open-source code SIESTA is used for all structural relaxations and energy calculations. ${ }^{5} \mathrm{~A} \mathrm{Ph}$ edge connected and basal connected CNT systems are considered with a CNT containing 5 unit cells and a large unit cell size such that periodic images are non-interacting. Generalized gradient approximation (GGA) with Perdew-Burke-Ernzerhof (PBE) exchange correlation functional is used with an energy cutoff of $500 \mathrm{Ry}$ for the real space grid. ${ }^{6}$ The broyden method is used for variable-cellrelaxation to obtain an energy minimized structure. ${ }^{7}$ Using the Monkhorst-Pack scheme, a 8 
$\times 8 \times 1$ k-point sampling of the Brillouin zone is used for electronic calculations. ${ }^{8}$
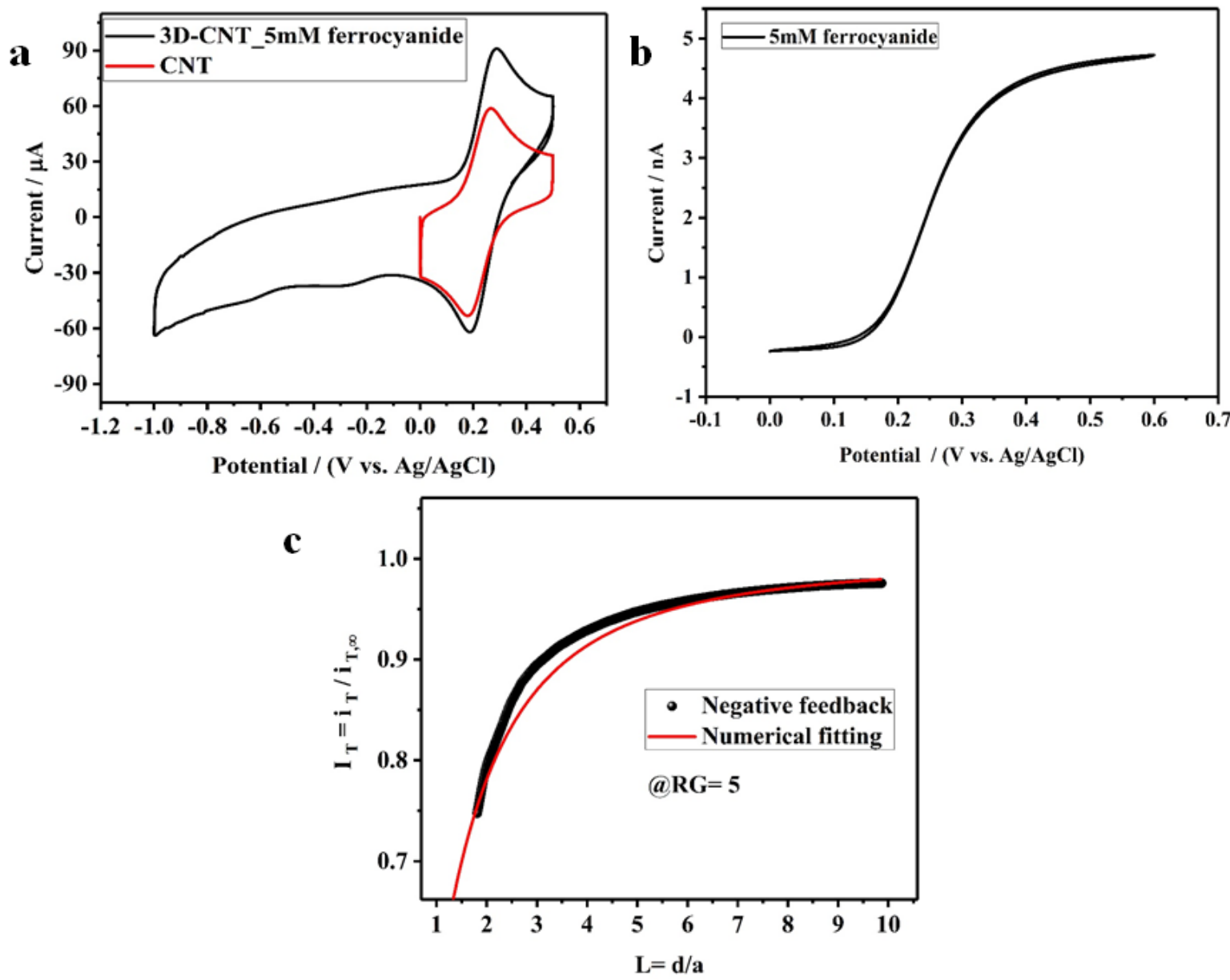

Figure S1. CV of (a) 3D-CNT and commercial CNT modified GCE in $5 \mathrm{mM}$ ferrocyanide solution at $50 \mathrm{mV} \mathrm{s}^{-1}$. (b) Pt-ultramicroelectrode ((UME) was characterized by recording CV in $5 \mathrm{mM}$ ferrocyanide solution at $50 \mathrm{mV} \mathrm{s}^{-1}$. (c)Probe approach curve (PAC) of Pt-UME was done in $5 \mathrm{mM}$ ferrocyanide solution at $0.5 \mathrm{~V}$ ( $\mathrm{vs} \mathrm{Ag} / \mathrm{AgCl}$ ) for oxidation of ferrocyanide to ferricyanide on insulating substrate. RG of Pt-UME was calculated from the fitting of experimental approach curve using euqation 2. 


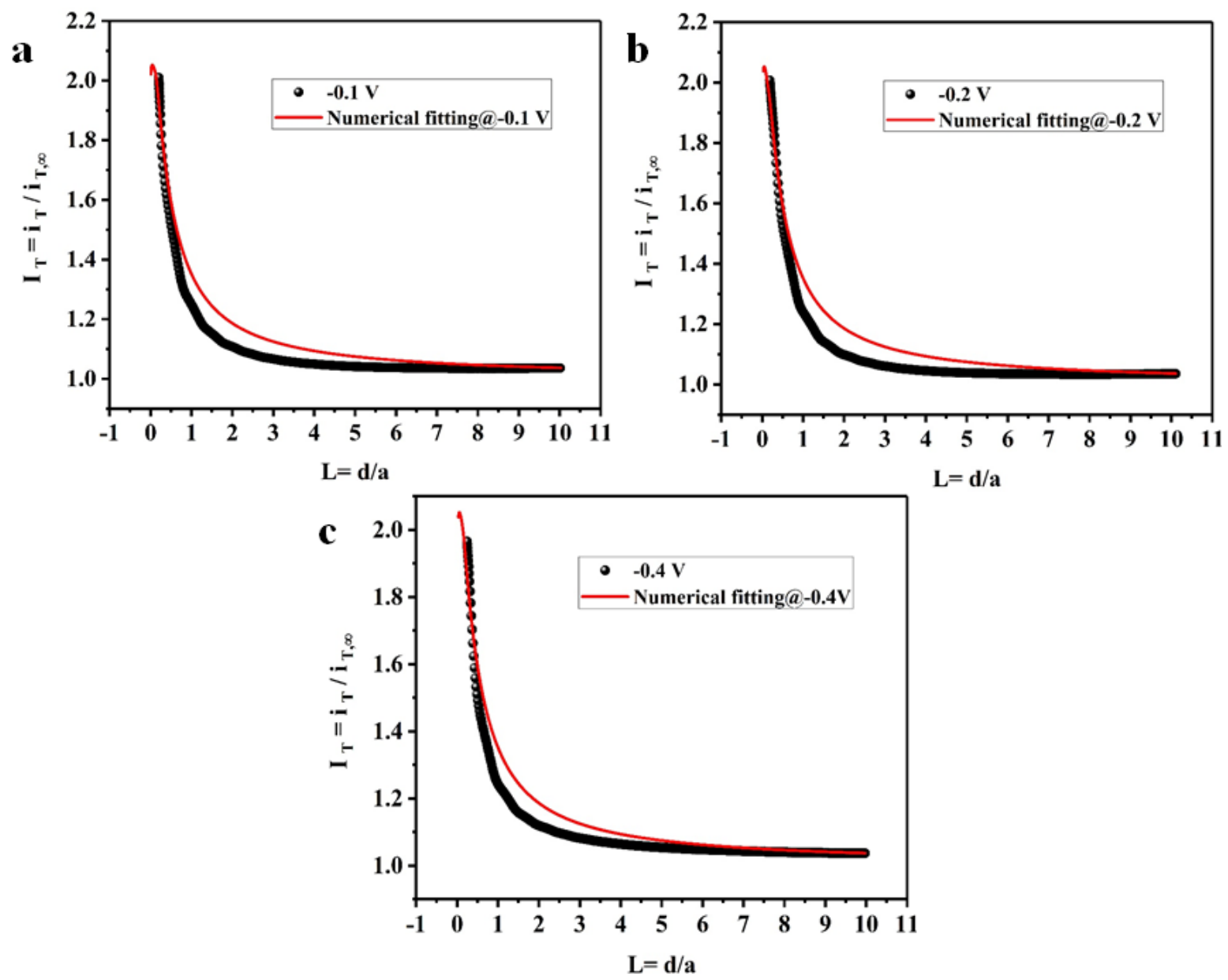

Figure S2. Probe approach curve (PAC) of 3D-CNT modified GCE using $5 \mathrm{mM}$ ferrocyanide solution as redox couple. Probe was approached to the substrate at different polarization potential (a) $-0.1 \mathrm{~V},(\mathrm{~b})-0.2 \mathrm{~V}$ and (c) $-0.4 \mathrm{~V}$ at fixed oxidation potential i.e. $0.5 \mathrm{~V}$ at the probe. Experimental probe approach curves were numerically fitted as shown in red line. These numerically fitting was carried out at different $\kappa$ values and from that effective rate constants were calculated. 

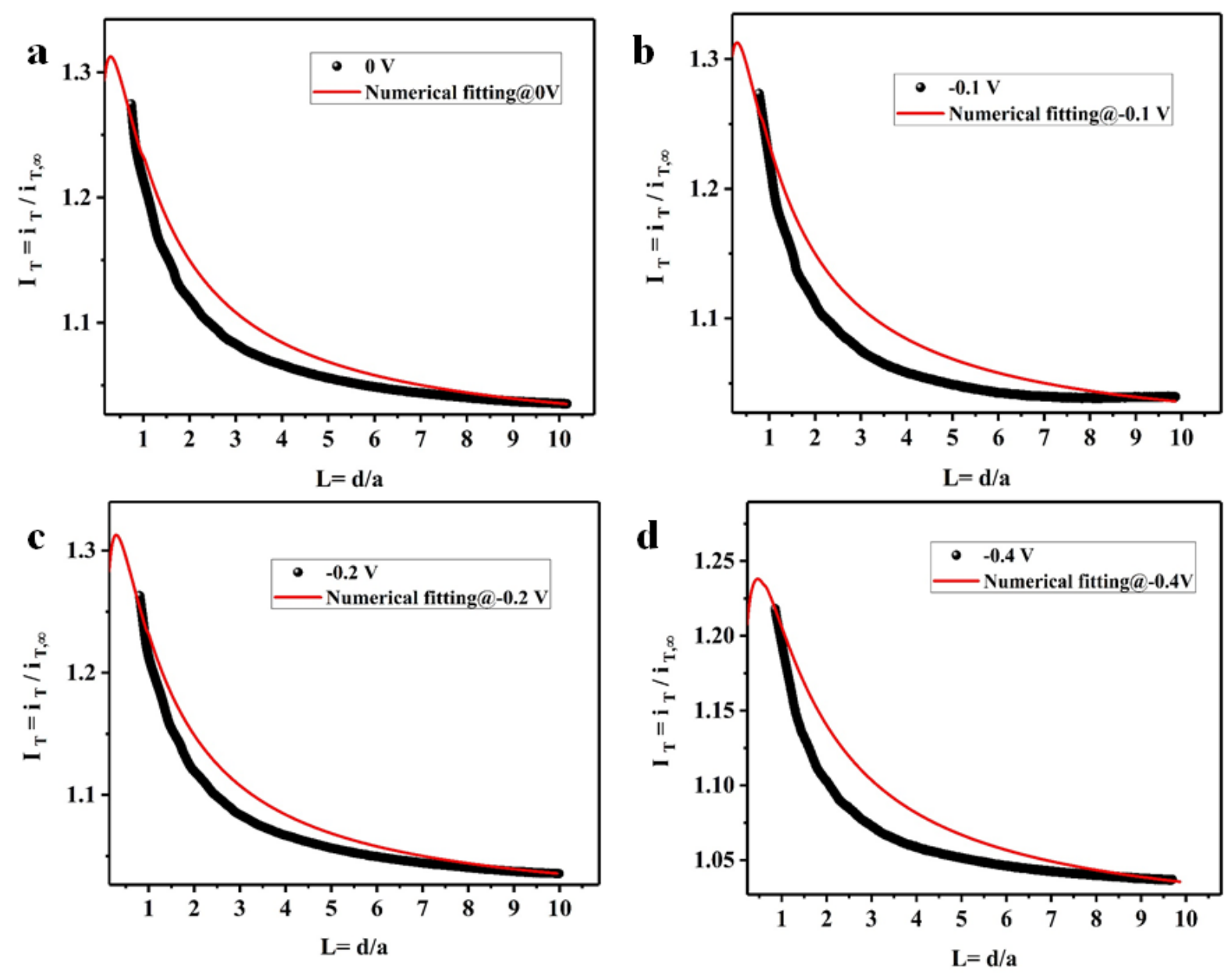

Figure S3. Probe approach curve (PAC) of CNT modified GCE using $5 \mathrm{mM}$ ferrocyanide solution as redox couple. Probe was approached to the substrate at different polarization potential (a) $0 \mathrm{~V}$, (b) $-0.1 \mathrm{~V}$, (c) $-0.2 \mathrm{~V}$ and (d) $-0.4 \mathrm{~V}$ at fixed oxidation potential i.e. $0.5 \mathrm{~V}$ at the probe. Experimental probe approach curves were numerically fitted as shown in red line. These numerically fitting was carried out at different $\kappa$ values and from that effective rate constants were calculated. 
(a)
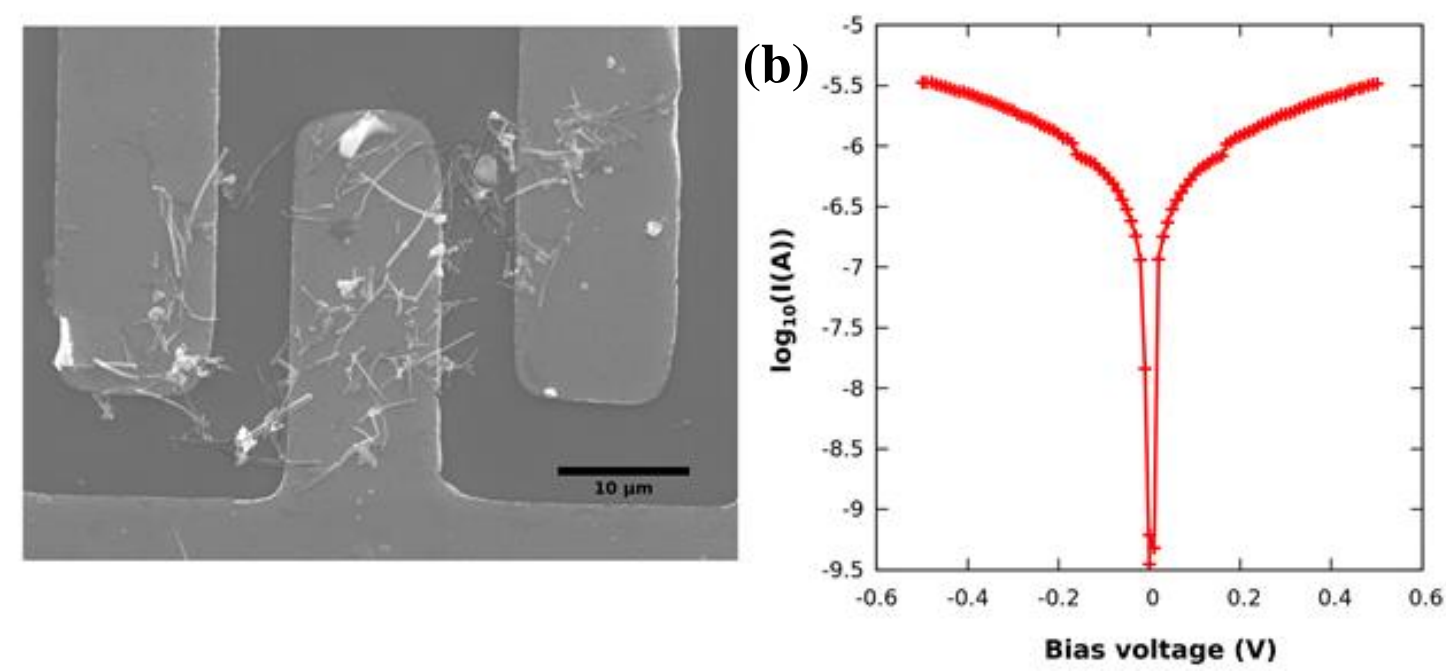

Figure S4. (a) A scanning electron microscope image of CNT-Ph lying over gold electrodes, and (b) the corresponding I-V plot showcasing the conductivity of CNT-Ph.

In order to monitor the conductivity of the materials the I-V characteristics were recorded. In Figure S4 the I-V characteristics of a CNT-Ph sample drop coated over gold pads (separated by $4 \mu \mathrm{m}$ ) along with the scanning electron microscope image are shown. Multiple CNTs, some of which are expected to contain $\mathrm{Ph}$ linkages span over the metal electrodes, and a conductance is seen due to this. The current (or conductance) seen here $(\sim 0.5 \mathrm{k} \Omega)$ is one order of magnitude lower than that observed in metallic MW-CNTs $(\sim 6 \mathrm{k} \Omega) .{ }^{9}$ Therefore, the covalent linkage can also help increase long range conductivity in the sample, apart from generating catalytically active sites. 


\section{REFERENCES}

1. Ozden, S.; Narayanan, T. N.; Tiwary, C. S.; Dong, P.; Hart, A. H.; Vajtai, R.; Ajayan, P. M., 3d Macroporous Solids from Chemically Cross-Linked Carbon Nanotubes. Small 2015, 11, 688-693.

2. Pal, S.; Narayanaru, S.; Kundu, B.; Sahoo, M.; Bawari, S.; Rao, D. K.; Nayak, S. K.; Pal, A. J.; Narayanan, T. N., Mechanistic Insight into Formate Production Via Co2 Reduction in C-C Coupled Carbon Nanotube Molecular Junctions. J. Phys. Chem. C 2018, 122, 2338523392.

3. Konopka, S.; McDuffie, B., Diffusion Coefficients of Ferri-and Ferrocyanide Ions in Aqueous Media, Using Twin-Electrode Thin-Layer Electrochemistry. Anal. Chem. 1970, 42, 1741-1746.

4. Cornut, R.; Lefrou, C., New Analytical Approximation of Feedback Approach Curves with a Microdisk Secm Tip and Irreversible Kinetic Reaction at the Substrate. J. Electroanal. Chem. 2008, 621, 178-184.

5. Soler, J. M.; Artacho, E.; Gale, J. D.; García, A.; Junquera, J.; Ordejón, P.; SánchezPortal, D., The Siesta Method for Ab Initio Order-N Materials Simulation. J. Phys. Condens. Matter 2002, 14, 2745.

6. Perdew, J. P.; Burke, K.; Ernzerhof, M., Generalized Gradient Approximation Made Simple. Phys. Rev. Lett. 1996, 77, 3865.

7. Johnson, D. D., Modified Broyden's Method for Accelerating Convergence in SelfConsistent Calculations. Phys. Rev. B 1988, 38, 12807.

8. Monkhorst, H. J.; Pack, J. D., Special Points for Brillouin-Zone Integrations. Phys. Rev. $B$ 1976, 13, 5188.

9. Frank, S.; Poncharal, P.; Wang, Z.; De Heer, W. A., Carbon Nanotube Quantum 
Resistors. Science 1998, 280, 1744-1746. 